\title{
Numerical evidence for universality in the excited instability spectrum of magnetically charged Reissner-Nordström black holes
}

\author{
Shahar Hod ${ }^{1,2, a}$ \\ ${ }^{1}$ The Ruppin Academic Center, 40250 Emeq Hefer, Israel \\ 2 The Hadassah Institute, 91010 Jerusalem, Israel
}

Received: 2 April 2015 / Accepted: 15 April 2015 / Published online: 30 April 2015

(C) The Author(s) 2015. This article is published with open access at Springerlink.com

\begin{abstract}
It is well known that the SU(2) ReissnerNordström black-hole solutions of the Einstein-Yang-Mills theory are characterized by an infinite set of unstable (imaginary) eigenvalues $\left\{\omega_{n}\left(T_{\mathrm{BH}}\right)\right\}_{n=0}^{n=\infty}$ (here $T_{\mathrm{BH}}$ is the black-hole temperature). In this paper we analyze the excited instability spectrum of these magnetically charged black holes. The numerical results suggest the existence of a universal behavior for these black-hole excited eigenvalues. In particular, we show that unstable eigenvalues in the regime $\omega_{n} \ll T_{\mathrm{BH}}$ are characterized, to a very good degree of accuracy, by the simple universal relation $\omega_{n}\left(r_{+}-r_{-}\right)=$constant, where $r_{ \pm}$are the horizon radii of the black hole.
\end{abstract}

\section{Introduction}

The familiar U(1) Reissner-Nordström spacetime is well known to describe a stable black-hole solution of the coupled Einstein-Maxwell equations [1,2] and the coupled EinsteinMaxwell-scalar equations [3-5]. Yasskin [6] has proved that the Einstein-Yang-Mills theory also admits an explicit black-hole solution which is described by the magnetically charged SU(2) Reissner-Nordström spacetime. However, the SU(2) Reissner-Nordström black-hole solution of the coupled Einstein-Yang-Mills equations is known to be unstable [7-10]. In fact, it was proved in $[11,12]$ that the magnetically charged Reissner-Nordström black-hole spacetime is characterized by an infinite family of unstable (growing in time) perturbation modes.

The recent numerical work of Rinne [13] has revealed that these unstable SU(2) Reissner-Nordström black-hole space-

a e-mail: shaharhod@gmail.com times play the role of approximate ${ }^{1}$ codimension-two intermediate attractors (that is, nonlinear critical solutions [14]) in the dynamical gravitational collapse of the Yang-Mills field. ${ }^{2}$ In particular, this interesting numerical study [13] has explicitly demonstrated that, during a near-critical evolution of the Yang-Mills field, the time spent in the vicinity of an unstable SU(2) Reissner-Nordström black-hole solution is characterized by the critical scaling law ${ }^{3}$

$\tau=$ const $-\gamma \ln \left|p-p^{*}\right|$.

Interestingly, the critical exponents of the scaling law (1) are directly related to the characteristic instability eigenvalues of the corresponding SU(2) Reissner-Nordström black holes [13]:

$\gamma=1 / \omega_{\text {instability }}$

It is therefore of physical interest to explore the instability spectrum $\left\{\omega_{n}\right\}_{n=0}^{n=\infty}$ of the SU(2) Reissner-Nordström black holes. Indeed, Rinne [13] has recently computed numerically the characteristic unstable eigenvalues of these magnetically charged black-hole solutions of the Einstein-Yang-Mills theory. ${ }^{4}$

\footnotetext{
${ }^{1}$ As emphasized in [13], the magnetically charged ReissnerNordström black-hole spacetime is only an approximate intermediate attractor because it is characterized by an infinite set of unstable (growing in time) modes.

2 This fact refers to type I and type III critical behaviors, see [13] for details.

${ }^{3}$ Here $\left|p-p^{*}\right|$ is a measure for the distance of the initial data from the threshold (critical) solution [14].

4 As emphasized above, the magnetically charged Reissner-Nordström black-hole solution of the Einstein-Yang-Mills theory is characterized by an infinite family of unstable perturbation modes [11,12]. Reference [13] provides, for the first time, detailed numerical results for the first three instability eigenvalues.
} 
In the present paper we shall analyze these numerically computed black-hole eigenvalues in an attempt to identify a possible hidden pattern which characterizes the black-hole instability spectrum. As we shall show below, the numerical results indeed suggest the existence of a universal behavior for these black-hole unstable eigenvalues.

\section{Description of the system}

The Reissner-Nordström black-hole solution of the EinsteinYang-Mills theory with unit magnetic charge is described by the line element [6]

$$
\begin{aligned}
\mathrm{d} s^{2}= & -\left(1-\frac{2 m}{r}\right) \mathrm{d} t^{2} \\
& +\left(1-\frac{2 m}{r}\right)^{-1} \mathrm{~d} r^{2}+r^{2}\left(\mathrm{~d} \theta^{2}+\sin ^{2} \theta \mathrm{d} \phi^{2}\right),
\end{aligned}
$$

where the mass function $m=m(r)$ is given by ${ }^{5}$

$m(r)=M-\frac{1}{2 r}$

The black-hole temperature is given by

$T_{\mathrm{BH}}=\frac{r_{+}-r_{-}}{4 \pi r_{+}^{2}}$

where

$r_{ \pm}=M \pm \sqrt{M^{2}-1}$

are the (outer and inner) horizons of the black hole.

Linearized perturbations $\xi(r) \mathrm{e}^{-i \omega t} 6$ of the magnetically charged black-hole spacetime are governed by the Schrödinger-like wave equation [15]

$\left\{\frac{\mathrm{d}^{2}}{\mathrm{~d} x^{2}}+\omega^{2}+\frac{1}{r^{2}}\left[1-\frac{2 m(r)}{r}\right]\right\} \xi=0$,

where the "tortoise" radial coordinate $x$ is defined by the relation $^{7}$

$\mathrm{d} x / \mathrm{d} r=[1-2 m(r) / r]^{-1}$.

Well-behaved (spatially bounded) perturbation modes are characterized by the boundary conditions

$\xi(x \rightarrow-\infty) \sim \mathrm{e}^{|\omega| x} \rightarrow 0$

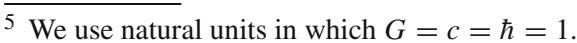

${ }^{6}$ Note that unstable (growing in time) modes are characterized by $\Im \omega>0$.

7 Note that the near-horizon limit $r \rightarrow r_{+}$corresponds to $x \rightarrow-\infty$, whereas the large- $r$ limit $r \rightarrow \infty$ corresponds to $x \rightarrow \infty$. and

$\xi(x \rightarrow \infty) \sim x e^{-|\omega| x} \rightarrow 0$,

where $\omega=i|\omega|$. As shown in $[9,11,12]$, these boundary conditions single out a discrete set of unstable $(\Im \omega>0)$ black-hole eigenvalues $\left\{\omega_{n}\left(r_{+}\right)\right\}_{n=0}^{n=\infty}$.

\section{Numerical evidence for universality in the excited instability spectrum}

Most recently, Rinne [13] computed numerically the first three instability eigenvalues which characterize the SU(2) Reissner-Nordström black-hole solutions of the coupled Einstein-Yang-Mills equations. We have examined these numerically computed eigenvalues in an attempt to reveal a possible hidden pattern which characterizes the black-hole instability spectrum.

In Table 1 we present the first excited instability eigenvalues $\left\{\omega_{1}\left(r_{+}\right)\right\}$of the magnetically charged SU(2) ReissnerNordström black holes. In particular, we display the dimensionless ratio $\omega_{1}\left(r_{+}\right) / \pi T_{\mathrm{BH}}$, where the black-hole temperature $T_{\mathrm{BH}}$ is given by (5). We also display the ratio between the dimensionless quantity $\omega_{1}\left(r_{+}\right) \times\left(r_{+}-r_{-}\right)$for generic SU(2) Reissner-Nordström black holes and the corresponding quantity $\omega_{1}\left(r_{+}=10\right) \times(10-1 / 10)$ for the weakly

Table 1 The instability eigenvalues of SU(2) Reissner-Nordström black holes. The data shown refers to the first excited eigenvalues $\left\{\omega_{1}\left(r_{+}\right)\right\}$of these magnetically charged black holes. We display the dimensionless ratio $\omega_{1}\left(r_{+}\right) / \pi T_{\mathrm{BH}}$, where $T_{\mathrm{BH}}$ is the black-hole temperature. Also shown is the ratio between the dimensionless quantity $\omega_{1}\left(r_{+}\right) \times\left(r_{+}-r_{-}\right)$for generic SU(2) Reissner-Nordström black holes and the corresponding quantity $\omega_{1}\left(r_{+}=10\right) \times(10-1 / 10)$ for the weakly magnetized Reissner-Nordström black hole with $r_{+}=10$ (see footnote 8 ). One finds that the instability eigenvalues in the regime $\omega_{1}\left(r_{+}\right) / \pi T_{\mathrm{BH}} \lesssim 0.1$ are characterized, to a good degree of accuracy, by the universal relation $\omega_{1}\left(r_{+}-r_{-}\right)=$constant

\begin{tabular}{lll}
\hline$r_{+}$ & $\omega_{1}\left(r_{+}\right) / \pi T_{\mathrm{BH}}$ & $\frac{\omega_{1}\left(r_{+}\right) \times\left(r_{+}-r_{-}\right)}{\omega_{1}\left(r_{+}=10\right) \times(10-1 / 10)}$ \\
\hline 9.0 & $9.86 \times 10^{-2}$ & 0.999 \\
8.0 & $9.91 \times 10^{-2}$ & 0.999 \\
7.0 & $9.99 \times 10^{-2}$ & 0.998 \\
6.0 & $1.01 \times 10^{-1}$ & 0.996 \\
5.0 & $1.04 \times 10^{-1}$ & 0.993 \\
4.0 & $1.08 \times 10^{-1}$ & 0.987 \\
3.0 & $1.18 \times 10^{-1}$ & 0.973 \\
2.0 & $1.58 \times 10^{-1}$ & 0.925 \\
1.5 & $2.57 \times 10^{-1}$ & 0.824 \\
1.2 & $6.04 \times 10^{-1}$ & 0.586 \\
\hline
\end{tabular}


Table 2 The instability eigenvalues of SU(2) Reissner-Nordström black holes. The data shown refers to the second excited eigenvalues $\left\{\omega_{2}\left(r_{+}\right)\right\}$of these magnetically charged black holes. We display the dimensionless ratio $\omega_{2}\left(r_{+}\right) / \pi T_{\mathrm{BH}}$, where $T_{\mathrm{BH}}$ is the black-hole temperature. Also shown is the ratio between the dimensionless quantity $\omega_{2}\left(r_{+}\right) \times\left(r_{+}-r_{-}\right)$for generic SU(2) Reissner-Nordström black holes and the corresponding quantity $\omega_{2}\left(r_{+}=10\right) \times(10-1 / 10)$ for the weakly magnetized Reissner-Nordström black hole with $r_{+}=10$ (see footnote 8). One finds that the instability eigenvalues in the regime $\omega_{2}\left(r_{+}\right) / T_{\mathrm{BH}} \ll 1$ are characterized, to a good degree of accuracy, by the universal relation $\omega_{2}\left(r_{+}-r_{-}\right)=$constant

\begin{tabular}{lll}
\hline$r_{+}$ & $\omega_{2}\left(r_{+}\right) / \pi T_{\mathrm{BH}}$ & $\frac{\omega_{2}\left(r_{+}\right) \times\left(r_{+}-r_{-}\right)}{\omega_{2}\left(r_{+}=10\right) \times(10-1 / 10)}$ \\
\hline 9.0 & $2.90 \times 10^{-3}$ & 1.011 \\
8.0 & $2.95 \times 10^{-3}$ & 1.021 \\
7.0 & $3.00 \times 10^{-3}$ & 1.029 \\
6.0 & $3.06 \times 10^{-3}$ & 1.034 \\
5.0 & $3.15 \times 10^{-3}$ & 1.038 \\
4.0 & $3.31 \times 10^{-3}$ & 1.040 \\
3.0 & $3.68 \times 10^{-3}$ & 1.041 \\
2.0 & $5.17 \times 10^{-3}$ & 1.039 \\
1.5 & $9.37 \times 10^{-3}$ & 1.034 \\
1.2 & $3.03 \times 10^{-2}$ & 1.011 \\
\hline
\end{tabular}

magnetized Reissner-Nordström black hole with $r_{+}=10 .^{8}$ Remarkably, the numerical data presented in Table 1 reveals that the black-hole instability eigenvalues in the regime $\omega_{1}\left(r_{+}\right) / T_{\mathrm{BH}} \ll 1$ are characterized, to a good degree of accuracy, by the universal relation ${ }^{9}$

$\omega_{1}\left(r_{+}-r_{-}\right)=\lambda_{1} ; \quad \lambda_{1}=$ constant

In order to support this intriguing finding, we display in Table 2 the second excited instability eigenvalues $\left\{\omega_{2}\left(r_{+}\right)\right\}$ of the SU(2) Reissner-Nordström black holes. Remarkably, the numerical data presented in Table 2 provide compelling evidence for the validity of the suggested universal behavior of the black-hole instability eigenvalues in the regime $\omega_{2}\left(r_{+}\right) / T_{\mathrm{BH}} \ll 1$. In particular, one finds ${ }^{10}$

$\omega_{2}\left(r_{+}-r_{-}\right)=\lambda_{2} ; \quad \lambda_{2}=$ constant

\section{Summary}

The U(1) Reissner-Nordström black holes are known to be stable within the framework of the coupled EinsteinMaxwell theory [1-5]. This stability property of the black

\footnotetext{
8 The weakly-magnetized SU(2) Reissner-Nordström black hole with horizon radius $r_{+}=10$ is the largest black-hole solution studied numerically in [13].

${ }^{9}$ It is worth emphasizing that, in the regime $\omega_{1}\left(r_{+}\right) / T_{\mathrm{BH}} \ll 1$, the value of $\lambda_{1}$ is almost independent of the black-hole horizon radius $r_{+}$.

${ }^{10} \mathrm{It}$ is worth emphasizing that, in the regime $\omega_{2}\left(r_{+}\right) / T_{\mathrm{BH}} \ll 1$, the value of $\lambda_{2}$ is almost independent of the black-hole horizon radius $r_{+}$.
}

holes manifests itself in the form of an infinite spectrum of damped quasi-normal resonances $[16,17]$. To the best of our knowledge, for generic U(1) Reissner-Nordström black holes, there is no simple universal formula which describes the infinite family of these damped black-hole quasi-normal resonances.

On the other hand, the SU(2) Reissner-Nordström black holes are known to be unstable within the framework of the coupled Einstein-Yang-Mills theory [7-10]. This instability property of the magnetically charged black holes manifests itself in the form of an infinite spectrum of exponentially growing black-hole resonances $[11,12]$. In this paper we have provided compelling numerical evidence that the infinite family of these unstable black-hole resonances can be described, to a very good degree of accuracy, by the simple universal formula

$\omega_{n}\left(r_{+}-r_{-}\right)=$constant $_{n}$ for $\omega_{n} \ll T_{\mathrm{BH}}$.

We believe that it would be highly interesting to find an analytical explanation for this numerically suggested universal behavior.

Acknowledgments This research is supported by the Carmel Science Foundation. I would like to thank Oliver Rinne for sharing with me his numerical data. I would also like to thank Yael Oren, Arbel M. Ongo, Ayelet B. Lata, and Alona B. Tea for helpful discussions.

Open Access This article is distributed under the terms of the Creative Commons Attribution 4.0 International License (http://creativecomm ons.org/licenses/by/4.0/), which permits unrestricted use, distribution, and reproduction in any medium, provided you give appropriate credit to the original author(s) and the source, provide a link to the Creative Commons license, and indicate if changes were made.

Funded by SCOAP 3 .

\section{References}

1. V. Moncrief, Phys. Rev. D 9, 2707 (1974)

2. V. Moncrief, Phys. Rev. D 10, 1057 (1974)

3. S. Hod, Phys. Lett. B 713, 505 (2012)

4. S. Hod, Phys. Lett. B 718, 1489 (2013). arXiv:1304.6474

5. S. Hod, Phys. Rev. D 91, 044047 (2015). arXiv:1504.00009

6. P.B. Yasskin, Phys. Rev. D 12, 108 (1975)

7. N. Straumann, Z.-H. Zhou, Phys. Lett. B 237, 353 (1990)

8. N. Straumann, Z.-H. Zhou, Phys. Lett. B 243, 33 (1990)

9. P. Bizoń, R.M. Wald, Phys. Lett. B 267, 173 (1991)

10. S. Hod, Phys. Lett. B 739, 157 (2014). arXiv: 1410.7406

11. P. Breitenlohner, P. Forgács, D. Maison, Nucl. Phys. B 383, 357 (1992)

12. P. Breitenlohner, P. Forgács, D. Maison, Nucl. Phys. B 442, 126 (1995)

13. O. Rinne, Phys. Rev. D 90, 124084 (2014)

14. C. Gundlach, J.M. Martín-García, Living Rev. Relat. 10 (2007) (see for an excellent review on the critical phenomena in gravitational collapse)

15. P. Bizoń, Phys. Lett B 259, 53 (1991)

16. E.W. Leaver, Phys. Rev. D 41, 2986 (1990)

17. S. Hod, Phys. Lett. B 710, 349 (2012). arXiv:1205.5087 